\title{
¿Cuál es el perfil de los pacientes en espera de nuevos tratamientos para la hepatitis C?
}

\author{
What is the Profile of Patients Waiting for New Hepatitis C Treatments?
}

João Alberto Martini Peruchi, MD, ${ }^{1}$ Emilia Tiemi Oshiro Bansho, MD, ${ }^{1}$ Débora Tonon, MD, ${ }^{1}$ Ariane Borgonovo, MD, ${ }^{1}$ Dariana Maggi, MD, ${ }^{1}$ Esther Buzaglo Dantas-Corrêa, MD, PhD, ${ }^{1}$ Leonardo de Lucca Schiavon, MD, PhD, ${ }^{1}$ Janaína Luz Narciso-Schiavon, MD, PhD. ${ }^{1}$

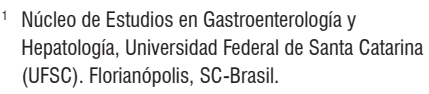

Fecha recibido: $04-05-15$ Fecha aceptado: 21-07-15

\section{Resumen}

Introducción: hasta hace poco, el tratamiento estándar de oro para la hepatitis $\mathrm{C}$ eran los interferones pegilados (Peg-IFN) en combinación con la ribavirina (RBV). Con la llegada de nuevos fármacos, se propuso evaluar los resultados del tratamiento y a los pacientes en espera de la nueva terapia.

Materiales y métodos: este estudio analítico transversal evaluó el resultado del tratamiento en individuos con hepatitis $\mathrm{C}$ crónica, y luego comparó a individuos que tienen experiencia en no responder al tratamiento basado en interferón (IFN) con individuos sin experiencia de tratamiento previo.

Resultados: el estudio incluyó 192 individuos. Entre 87 pacientes sometidos a tratamiento, se observaron bajas tasas de respuesta viral sostenida (RVS). La comparación de los 105 pacientes no tratados previamente y los 87 que habían recibido tratamiento con IFN previamente evidenció que entre los pacientes en espera de nuevas terapias, los individuos sin tratamiento previo presentaron mayor proporción de genotipo 1 (68\% frente a 49\%; $p=0,028)$, menores niveles de ALT (91,1 $\pm 73,0$ frente a 126,0 $\pm 73,40 \mathrm{U} / \mathrm{L} ; p=017)$, de AST $(70,1 \pm 51,3$ frente a $89,7 \pm 47,40 \mathrm{U} / \mathrm{L} ; p=050)$, de GGT $(85,3 \pm 85,1$ frente a 148,4 $\pm 123,9 \mathrm{U} / \mathrm{L} ; p=0,007)$ y menor proporción de fibrosis significativa (24,3 frente a 83,$3 ; p<0,001)$.

Conclusiones: las tasas de RVS fueron bajas. La mayoría de posibles candidatos para el tratamiento por VHC no lo han tenido y son de genótipo-1 con histología leve.

\section{Palabras clave}

Hepatitis C, resultado del tratamiento, interferón alfa.

\begin{abstract}
Introduction: Until recently, treatment with a combination of pegylated interferons (Peg-IFN) and ribavirin (RBV) was the gold standard treatment for hepatitis $\mathrm{C}$. In anticipation of the arrival of new drugs, we evaluate current treatment outcomes and patients waiting for the new therapy.

Materials and Methods: This cross-sectional analytical study evaluated treatment outcomes among chronic hepatitis $C$ patients, and then compared chronic non-responders and treatment naïve patients who were given interferon based-treatment.

Results: The study included 192 individuals among whom were 87 patients who received treatment. Among treated patients, we observed low rates of sustained viral response. A comparison of 105 treatmentnaïve patients and 87 who had previously received IFN treatment showed that among patients waiting for new therapies, naïve individuals presented a higher proportion of genotype $1(68 \%$ vs. $49 \% ; p=0.028)$ than did previously treated patients, lower ALT ( $91.1 \pm 73.0$ vs. $126.0 \pm 73.40 \mathrm{U} / \mathrm{L} ; p=017)$, lower AST $(70.1 \pm 51.3$ vs. $89.7 \pm 47.40 \mathrm{U} / \mathrm{L} ; p=050)$, lower GGT ( $85.3 \pm 85.1$ vs. $148.4 \pm 123.9 \mathrm{U} / \mathrm{L} ; p=0.007)$ levels and a lower proportion of significant fibrosis $(24.3 \%$ vs. $83.3 \%$; $p<0.001)$.

Conclusions: SVR rates were low. Among potential candidates for HCV treatment, the majority are naïve, genotype-1 with mild histology.
\end{abstract}

\author{
Keywords \\ Hepatitis C, treatment outcome, interferon-alpha.
}




\section{INTRODUCCIÓN}

Hasta hace poco, el interferón pegilado alfa (Peg-IFN) en combinación con la ribavirina era el tratamiento estándar más usado para la hepatitis C. En 2011, el telaprevir y boceprevir fueron autorizados para manejar la infección por virus de la hepatitis C (VHC) de genotipo 1; estos 2 medicamentos son antivirales de primera de ola, de primera generación de acción directa. Recientemente han surgido nuevos fármacos que producen menos efectos secundarios y tratamientos de menor duración, especialmente en los países desarrollados; estos han cambiado la atención estándar por la terapia triple (1). Los inhibidores de proteasa de segunda y tercera generación no están ampliamente disponibles en Brasil, y el esquema dual con interferón estándar (IFN) sigue siendo recomendado por el Ministerio Nacional de Salud para los pacientes no cirróticos con VHC de genotipos 2 o 3 . Con la llegada de nuevos medicamentos se busca evaluar el perfil de pacientes que han tenido experiencia previa de tratamiento y de otros que no han sido tratados previamente que están en espera de este proceso. El objetivo del estudio fue determinar el perfil clínico de pacientes tratados previamente y de pacientes no tratados previamente que esperan la nueva terapia para el VHC.

\section{MATERIALES Y MÉTODOS}

Este estudio analítico transversal evaluó portadores del VHC (adultos) en la clínica ambulatoria de gastroenterología y hepatología de un hospital universitario público, entre enero y mayo de 2014. Algunos pacientes se excluyeron por las siguientes razones: registro insuficiente de datos clínicos, negativa a participar en el estudio, hepatitis $\mathrm{C}$ aguda, ARN-VHC negativo, o tratamiento basado en IFN en curso. En visita de rutina para pacientes externos se invitó a las personas a participar en el estudio y a firmar el formulario de consentimiento; de los registros de historias clínicas se obtuvieron datos clínicos, de laboratorio e histológicos, se definió a los pacientes con VHC como aquellos con ácido ribonucleico (ARN) del VHC detectable por la reacción en cadena de la polimerasa (PCR). Las variables clínicas y de laboratorio analizadas fueron género masculino, edad, color de piel, ingesta de alcohol superior a $40 \mathrm{~g}$ por día, creatinina, plaquetas, alanina aminotransferasa (ALT), aspartato aminotransferasa (AST), gamma-glutamiltransferasa (GGT), bilirrubina directa, albúmina, índice internacional normalizado, genotipo del VHC y carga viral. La respuesta virológica sostenida (RVS) se definió como ARN del VHC indetectable por 24 semanas después de finalizar el tratamiento. Las características histológicas fueron analizadas utilizando el sistema de puntuación grupal METAVIR. La fibrosis se defi- nió en una escala de F0 a F4 de la siguiente manera: F0 = no fibrosis, $\mathrm{F} 1$ = fibrosis portal sin tabiques, $\mathrm{F} 2$ = pocos tabiques, F3 = numerosos tabiques sin cirrosis, y F4 = cirrosis; la fibrosis significativa se definió por la presencia de las etapas METAVIR F2, F3, o F4, y la ocurrencia de las etapas F3 o F4 caracterizaron a la fibrosis avanzada (2).

Los primeros tratamientos de los pacientes fueron evaluados retrospectivamente. Ellos recibieron IFN, Peg-IFN, o inhibidores de proteasa de primera generación telaprevir o boceprevir (PI) más Peg-IFN. Todos los regímenes de tratamiento incluyeron ribavirina basada en el peso durante 24 o 48 semanas, dependiendo del genotipo del VHC o del enfoque individual (cirrosis, por ejemplo). Las variables continuas con distribución normal fueron expresadas como media y desviación estándar y comparadas mediante la prueba $t$ de Student. Las variables categóricas fueron representadas por frecuencia (\%) y analizadas mediante la prueba chi-cuadrado, asociación chi-cuadrado lineal por lineal, o con la prueba exacta de Fisher cuando fue necesario. Los valores de $p$ inferiores a 0,05 fueron considerados estadísticamente significativos.

Se realizó un análisis bivariado para identificar las variables asociadas con los pacientes no tratados previamente. Las variables con valores de $p$ inferior a 0,20 se incluyeron en el análisis multivariado. Se realizó un análisis de regresión logística binaria (enter) para identificar variables asociadas de forma independiente con los pacientes no tratados previamente. Todas las pruebas se realizaron con el software Paquete Estadístico IBM para Ciencias Sociales, versión 17.0 (SPSS Statistics, Chicago, Illinois, EE.UU.). El protocolo del estudio se adhiere a los principios éticos de la Declaración de Helsinki y fue aprobado por el comité local de ética (número 832.915).

\section{RESULTADOS}

Entre enero y mayo de 2014, nuestra institución trató y evaluó para inscripción a 228 pacientes con VHC crónica; 36 pacientes fueron excluidos por las siguientes razones: 11 resultaron repetidamente negativos al VHC-ARN, 4 estaban bajo tratamiento, 4 tenían datos incompletos en las historias clínicas, y 4 tenían enfermedad hepática avanzada (figura 1). El estudio incluyó a 192 personas con VHC de una edad promedio de 51,8 $\pm 11,8$ años (media de 52,0); $47,4 \%$ fueron hombres y el $94,7 \%$ declararon ser de raza blanca (caucásicos). El genotipo estuvo disponible para 165 pacientes y se distribuyó de la siguiente manera: el genotipo $1=60 \%$; genotipo $2=3 \%$; genotipo $3=37 \%$; entre los sujetos con genotipo 1 , el $15,6 \%$ fueron genotipo 1a, genotipo 1 b $6,7 \%$ y el laboratorio no informó el subtipo del $77,8 \%$. Se observó fibrosis avanzada en el $45,9 \%$ de la muestra y el 
$33 \%$ presentó cirrosis. En lo que respecta al tratamiento del VHC, 105 pacientes eran nuevos al tratamiento y 87 habían recibido tratamiento con IFN previamente.

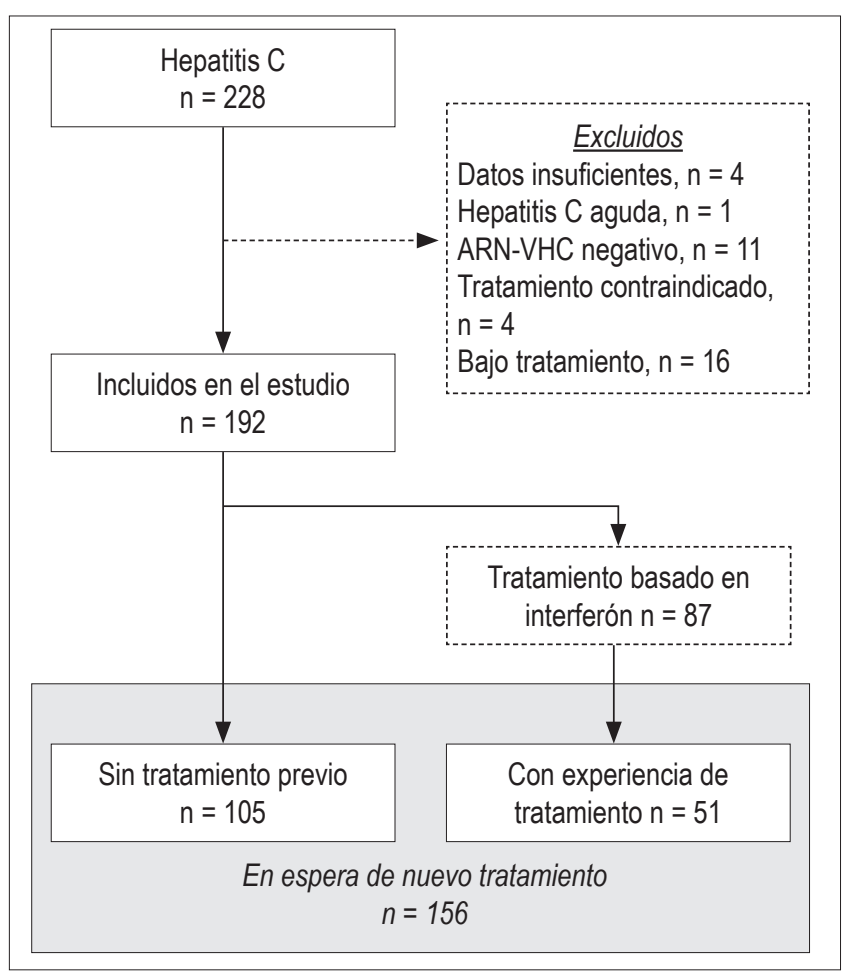

Figura 1. Diagrama de flujo de posibles candidatos para la participación en el estudio, incluidos los criterios de exclusión y los individuos.

De los 87 individuos sometidos a tratamiento basado en interferón, el 84,5\% tenía más de 40 años, el 51,8\% era de genotipo 1 , el $60,3 \%$ presentó fibrosis avanzada ( $45 \%$ era cirrótico), y el $54,5 \%$ presentó carga viral $\geq 800000,00$. El IFN estándar fue administrado a $14,9 \%$, Peg-IFN a $73,6 \%$, y PI al $11,5 \%$ de los pacientes $(\mathrm{p}=0,136)$. De los 10 pacientes tratados con PI, 7 recibieron telaprevir y 3 recibieron boceprevir. La RVS general se obtuvo para el $50 \%$ de los pacientes tratados: $56 \%$ de genotipo 1 y $42,3 \%$ de no genotipo 1 (p $=0,328$ ); entre los de no genotipo 1 , el $22,2 \%$ obtuvo SVR con terapia convencional IFN + ribavirina y el $52,9 \%$ obtuvo SVR con Peg-IFN + ribavirina. Entre los de genotipo 1, el $57,1 \%$ obtuvo RVS con Peg-IFN + ribavirina y el 50,0\% obtuvo SVR con PI + Peg-IFN + ribavirina (figura 2).

Veinte pacientes fueron sometidos a un segundo régimen de tratamiento, todos los cuales tenían más de 40 años de edad y presentó fibrosis avanzada; $40 \%$ eran hombres y el 42,1\% eran del genotipo 1. 1 (5\%) recibió IFN estándar, 13 (65\%) recibieron Peg-IFN, y 6 (30\%) fueron tratados con PI. Las tasas de RVS del segundo tratamiento estuvieron disponibles para 16 pacientes e indican un 43,8\% de RVS: $27,3 \%$ para Peg-IFN y un $80 \%$ para PI $(\mathrm{p}=0,106)$. Dos pacientes fueron sometidos a un tercer régimen de tratamiento con Peg-IFN durante 72 semanas y ambos lograron RVS.

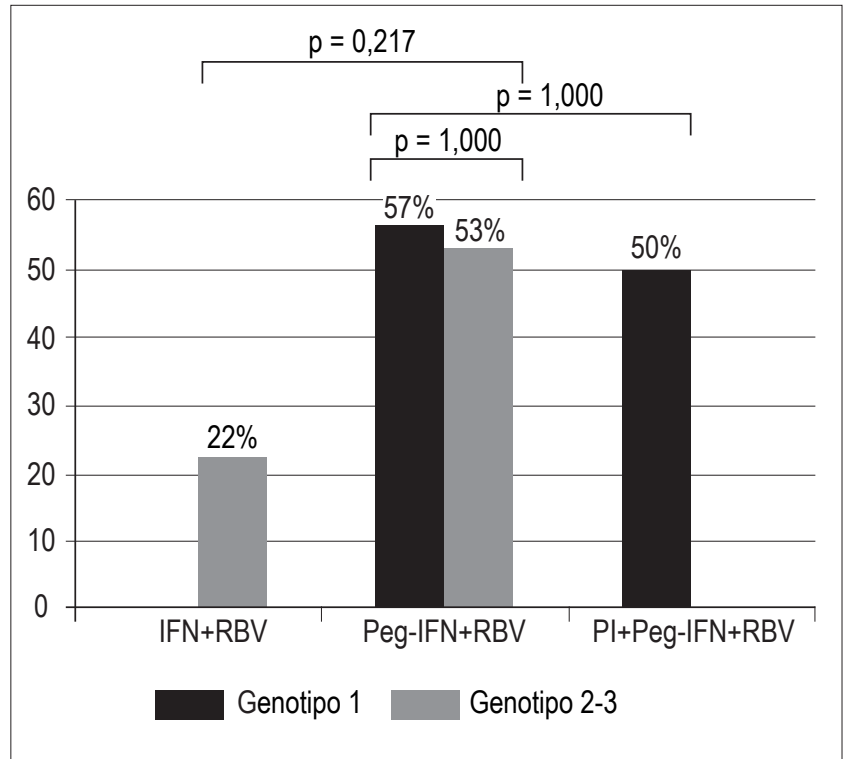

Figura 2. Tasas de respuesta virológica sostenida de 87 pacientes con hepatitis $\mathrm{C}$ crónica tratados con regímenes basados en interferón. IFN: interferón convencional, RBV: ribavirina; Peg-IFN: interferón pegilado; IP: inhibidor de proteasa.

De los 156 pacientes candidatos potenciales para el tratamiento del VHC, 105 no habían sido tratados previamente y 51 ya tenían experiencia de tratamiento (tabla 1 ). Casi $2 / 3$ de los pacientes no tratados previamente eran de genotipo $1 \mathrm{y}$ presentaron cargas virales altas en promedio; sin embargo, solo 1/4 de ellos presentó fibrosis avanzada; la mitad de los pacientes con experiencia de tratamiento previo presentó VHC genotipo 1 y $3 / 4$ tenían fibrosis avanzada. Los pacientes con experiencia previa presentaron niveles promedio de ALT, AST y GGT más elevados en comparación con los pacientes no tratados previamente (tabla 1). En el análisis multivariado se incluyeron las siguientes variables: genotipo no 1, ALT, AST, GGT, y fibrosis no significativa; el análisis de regresión logística binaria (enter) reveló que el genotipo no $1(\mathrm{OR}=1,006$; IC $95 \%$ con rango de 1,001 a 1,$011 ; \mathrm{p}=0,031$ ) y la fibrosis no significativa se asociaron independientemente con los pacientes sin tratamiento previo ( $\mathrm{OR}=0,071$; IC $95 \%$ con rango de 0,019-0,271; $\mathrm{p}<0,001)$.

\section{DISCUSIÓN}

En lo que respecta a las variables de edad, sexo, etnia y genotipo, la presente muestra de estudio no difiere de lo que se ha observado en Brasil y en otros países. Es de destacar que casi el $100 \%$ de los pacientes se autodeclararon 
Tabla 1. Características clínicas y de laboratorio de 156 individuos candidatos potenciales a tratamiento contra la infección crónica por VHC, de acuerdo con la experiencia previa de tratamiento basado en interferón.

\begin{tabular}{|c|c|c|c|c|}
\hline Características & $\begin{array}{c}\text { Todos } n=156 \\
(100 \%)\end{array}$ & $\begin{array}{c}\text { Sin tratamiento previo } \\
\mathrm{n}=105(65,4 \%)\end{array}$ & $\begin{array}{l}\text { Con experiencia de tratamiento } \\
n=51(34,6 \%)\end{array}$ & $p$ \\
\hline Sexo masculino $(\mathrm{n}, \%)$ & $72(46,2)$ & $32(50,0)$ & $24(47,1)$ & $0,874 \times$ \\
\hline Edad $\geq 40$ años $(n, \%)$ & $130(83,3)$ & $85(81,0)$ & $45(88,2)$ & $0,252^{x}$ \\
\hline Raza blanca (n, \%) & $126(94,0)$ & $83(92,2)$ & $43(97,7)$ & $0,272^{f}$ \\
\hline Ingesta de alcohol >40 g/d (n, \% $)^{\dagger}$ & $42(35,3)$ & $25(32,5)$ & $17(40,5)$ & $0,382^{f}$ \\
\hline Genotipo $1(\mathrm{n}, \%)^{+t}$ & $80(61,1)$ & $56(68,3)$ & $24(49,0)$ & $0,028 \times$ \\
\hline HBsAg positivo (n, \%) & $5(3,4)$ & $4(4,1)$ & $1(2,0)$ & $0,663^{f}$ \\
\hline VIH positivo (n, \%) & $2(1,4)$ & $2(2,1)$ & $0(0,0)$ & $0,548^{f}$ \\
\hline Creatinina $(\mathrm{mg} / \mathrm{dL})^{*}$ & $0,9 \pm 0,2$ & $0,9 \pm 0,3$ & $0,8 \pm 0,2$ & $0,285^{t}$ \\
\hline Plaquetas $\left(/ \mathrm{mm}^{3}\right)^{*}$ & $186795,0 \pm 82958,8$ & $197142,9 \pm 74802,0$ & $169486,5 \pm 95616,6$ & $0,079^{t}$ \\
\hline $\operatorname{ALT}(U / L)^{*}$ & $107,4 \pm 78,0$ & $91,1 \pm 73,0$ & $126,0 \pm 73,4$ & $0,017^{t}$ \\
\hline $\operatorname{AST}(\mathrm{U} / \mathrm{L})^{*}$ & $78,0 \pm 51,9$ & $70,1 \pm 51,3$ & $89,7 \pm 47,4$ & $0,050^{t}$ \\
\hline GGT (U/L)* & $101,8 \pm 95,7$ & $85,3 \pm 85,1$ & $148,4 \pm 123,9$ & $0,007^{t}$ \\
\hline Albúmina $(\mathrm{g} / \mathrm{dL})^{*}$ & $3,9 \pm 0,5$ & $3,8 \pm 0,5$ & $3,9 \pm 0,4$ & $0,387^{t}$ \\
\hline $\mathrm{INR}^{*}$ & $1,1 \pm 0,1$ & $1,1 \pm 0,2$ & $1,1 \pm 0,1$ & $0,385^{t}$ \\
\hline Carga viral $(\mathrm{Ul} / \mathrm{mL})^{+t+}$ & $2192795 \pm 3676043$ & $1978642 \pm 3214561$ & $3063141 \pm 4962591$ & $0,288^{t}$ \\
\hline Fibrosis significativatttt & $63(54,8)$ & $28(24,3)$ & $35(83,3)$ & $<0,001$ \\
\hline
\end{tabular}

AST: aspartato aminotransferasa; GGT: gamma glutamiltransferasa; INR: índice normalizado internacional; fibrosis significativa: METAVIR F2, F3 o F4; * media \pm desviación estándar. $x$ : prueba de Chi-cuadrado; $t$ : prueba $t$ de Student; $f$ : prueba exacta de Fisher. Información disponible en: $+119,++131,+++67 \mathrm{y}++++115$ individuos.

como de raza blanca, especialmente en un país de mestizaje como Brasil; en el sur de este país, la colonización fue predominantemente de origen europeo (3) y la mayoría de la población es de raza blanca, según lo informado por otros estudios brasileños del sur que evalúan individuos con VHC (92\%-99\%) (4, 5).

Desde el año 2000, se ha sabido bien que para los pacientes con hepatitis $\mathrm{C}$ crónica un régimen de Peg-IFN + RBV es más eficaz que un régimen de IFN + RBV administrado 3 veces por semana ( $39 \%$ versus. $19 \%, p=0,001)(6)$. En los pacientes con infección VHC de genotipo 1 , las tasas de RVS correspondientes fueron entre $42 \%$ a $46 \%(7,8)$, mientras que la tasa para los pacientes con infecciones de genotipos 2 y 3 fue de alrededor del $80 \%$, independientemente del régimen de IFN (7). Esta fue una de las justificaciones utilizadas en Brasil para mantener el esquema con IFN + RBV como tratamiento de primera línea para pacientes no cirróticos con genotipos 2 y 3 . Respecto de los individuos de genotipo no 1 , se observaron tasas de RVS similares entre los que recibieron IFN + RBV y los que recibieron Peg-IFN + RBV (figura 1), probablemente debido al pequeño número de pacientes evaluados con genotipo no $1(n=24)$. Sobre lo antes mencionado, hay que señalar el hecho de que en Brasil los pacientes de genotipo no 1 solo pueden recibir Peg-IFN si presentan fibrosis avanzada, los otros solo pueden ser tratados con IFN biosimilar estándar, el cual es más barato en términos de salud pública pero ha demostrado ser peor que el Peg-IFN en eficacia. Vigani y colaboradores han demostrado que el Peg-IFN + RBV está asociado con mayores tasas de RVS cuando se compara con el IFN biosimilar estándar independientemente de la etapa de la fibrosis $(79,3 \%$ versus $49,1 \%, \mathrm{p}=0,0001)(9)$.

La RVS para individuos con genotipo 1 que recibieron Peg-IFN + RBV fue 57,1\%, la cual es superior a los valores reportados en otros estudios brasileños de $43 \%$ a $52 \%$ (10, 11) e inferior a la de los ensayos clínicos aleatorios internacionales. En un estudio retrospectivo de pacientes brasileños del sur con hepatitis crónica e infección VHC genotipo 1, la RVS se alcanzó en solo el 35,3\% de los pacientes (114/323), aunque esto puede deberse a que una gran proporción de ellos presentaron fibrosis avanzada (F3 / F4 = 74\%) (12). En el presente estudio, el 52\% de los pacientes con genotipo 1 presentó fibrosis avanzada, una proporción más alta que la reportada en los registros de estudio, donde las tasas de RVS para Peg-IFN + ribavirina variaron entre $42 \%$ y $52 \%$, y entre $24 \%$ y $55 \%$ presentaron fibrosis avanzada $(7,8)$.

Se sabe que entre las variables que más interfieren con la SVR están la edad, raza, sexo, índice de masa corporal, carga 
viral, y grado de fibrosis $(1,7,13)$. Cuando se evaluaron las variables asociadas con mejores tasas de RVS, hubo una tendencia a bajar solo la carga viral (RVS $1172172 \pm 1342722$ versus. no-RVS $4079651 \pm 5275628 ; \mathrm{p}=0,054)$, probablemente debido al pequeño número de pacientes incluidos en los subgrupos de diferentes regímenes de tratamiento. Aunque hubo una preponderancia de individuos de raza blanca en esta muestra, muchas de las variables conocidas como asociadas con la RVS fueron desfavorables en la mayoría de los pacientes que recibieron tratamiento: edad, genotipo (97\% de la muestra presentó genotipo 1 o 3), fibrosis avanzada y carga viral, y estos hallazgos, aunque no han demostrado importancia estadística (excepto la carga viral), no pueden ser ignorados como una posible influencia en las bajas tasas de RVS descritas en esta muestra.

Solo 10 pacientes en el presente estudio fueron tratados con PI, y por lo tanto no se puede saber si las tasas de RVS representan los resultados reales de nuestra clínica. La tasa de $50 \%$ de RVS encontrada en este grupo está muy por debajo de las tasas de los primeros ensayos (14); en la fase III de los ensayos clínicos, las tasas de RVS en pacientes sin tratamiento previo se reportaron en $66 \%$ y $75 \%$ para pacientes tratados con regímenes basados en boceprevir y telaprevir, respectivamente $(15,16)$. En los estudios registrados, entre $20 \%$ y $50 \%$ de los pacientes estudiados presentaron fibrosis avanzada $(15,16)$. En el ámbito clínico español de tratamiento con boceprevir PI, un total de $80 \%$ tenía fibrosis F3/F4 y la negativación del ARN fue 48,8\% en la semana 8 (17). En los EE.UU., un grupo real de 200 pacientes demostró $53 \%$ de RVS para telaprevir y $40 \%$ de boceprevir (18).

La alta eficacia y seguridad de nuevos fármacos dan esperanza al tratamiento universal de las personas infectadas con el VHC; sin embargo, el alto costo del tratamiento masivo puede dificultar su uso. Actualmente, hay 4 medicamentos aprobados por la Agencia Nacional de Vigilancia Sanitaria (ANVISA), compañía que regula los medicamentos en Brasil: sofosbuvir, daclatasvir, simeprevir y «3D» (ABT-450, ritonavir, ombitasvir y dasabuvir), pero ninguno de ellos está todavía disponible para el tratamiento en el Sistema de Salud Pública (SUS). El enfoque terapéutico probable está bajo consulta pública y los médicos no saben qué estará disponible en un futuro próximo y quién será tratado de acuerdo con las directrices del gobierno. Los precios se estiman entre $\$ 2.000 .00$ y $\$ 2.500 .00$ dólares estadounidenses per cápita por mes, lo cual puede restringir la selección de pacientes a los que no responden y a aquellos con fibrosis avanzada (19). En el futuro, la llegada de nuevos fármacos al mercado puede que baje los precios y facilite el acceso de los pacientes que están en necesidad de tratamiento a los medicamentos sin restricciones.
Es importante reconocer algunas limitaciones de este análisis. El defecto principal y más evidente de los estudios de un solo centro es su potencialmente limitada validez externa, aunque estos permiten estudios más grandes, multicéntricos, que deben ser planeados y apoyados adecuadamente; este estudio se realizó en un servicio especializado de referencia para el tratamiento de la hepatitis $\mathrm{C}$ y la muestra estudiada es comparable con otras poblaciones en el mundo. En segundo lugar, el número relativamente pequeño de pacientes incluidos podría limitar la interpretación de los resultados; sin embargo, los datos recogidos representan la realidad de la práctica clínica.

En conclusión, el tratamiento doble con Peg-IFN y RBV es el régimen basado en IFN más comúnmente administrado en los pacientes con VHC, y exhibe tasas de RVS similares a las de otros esquemas; en este estudio, las tasas de RVS demostraron ser inferiores a las reportadas en la literatura. Entre los posibles candidatos al tratamiento del VHC, la mayoría fueron los no tratados previamente de genotipo 1 y de hallazgos histológicos leves. La mitad de los pacientes con experiencia de tratamiento previo fueron de genotipo 1, y la mayoría presentó fibrosis avanzada.

\section{Fuentes de financiación}

Los autores no contaron con apoyo financiero.

\section{Conflicto de intereses}

Los autores declaran no tener conflictos de interés.

\section{Reconocimientos}

Trabajo presentado como requisito para la obtención del título de Doctor en Medicina (MD) de la Universidad Federal de Santa Catarina (UFSC).

\section{REFERENCIAS}

1. European Association for the Study of the Liver. EASL recommendations on treatment of hepatitis C 2014. J Hepatol. 2014;61:373-95.

2. Bedossa P, Poynard T. An algorithm for the grading of activity in chronic hepatitis C. The METAVIR Cooperative Study Group. Hepatology. 1996;24:289-93.

3. Pena SD, Di Pietro G, Fuchshuber-Moraes M, Genro JP, Hutz MH, Kehdy Fde S, et al. The genomic ancestry of individuals from different geographical regions of Brazil is more uniform than expected. PLoS One. 2011;6:e17063.

4. Martins T, Machado DF, Schuelter-Trevisol F, Trevisol DJ, Vieira e Silva RA, Narciso-Schiavon JL, et al. Prevalence and factors associated with $\mathrm{HCV}$ infection among elderly indi- 
viduals in a southern Brazilian city. Rev Soc Bras Med Trop. 2013;46:281-7.

5. Silveira L, Schiavon L de L, Silva KP, Lopes TB, Zaccaron Mda R, Narciso-Schiavon JL. Clinical and epidemiological profile of blood donors with positive serology for viral hepatitis in southern Brazil. Rev Soc Bras Med Trop. 2011;44:269-73.

6. Zeuzem S, Feinman SV, Rasenack J, Heathcote EJ, Lai MY, Gane E, et al. Peginterferon alfa-2a in patients with chronic hepatitis C. N Engl J Med. 2000;343:1666-72.

7. Manns MP, McHutchison JG, Gordon SC, Rustgi VK, Shiffman M, Reindollar R, et al. Peginterferon alfa-2b plus ribavirin compared with interferon alfa- $2 b$ plus ribavirin for initial treatment of chronic hepatitis C: a randomised trial. Lancet. 2001;358:958-65.

8. Hadziyannis SJ, Sette H Jr., Morgan TR, Balan V, Diago M, Marcellin P, et al. Peginterferon-alpha2a and ribavirin combination therapy in chronic hepatitis $\mathrm{C}$ : a randomized study of treatment duration and ribavirin dose. Ann Intern Med. 2004; 140:346-55.

9. Vigani AG, Goncales ES, Pavan MH, Genari F, Tozzo R, Lazarini MS, et al. Therapeutic effectiveness of biosimilar standard interferon versus pegylated interferon for chronic hepatitis C genotypes 2 or 3. Braz J Infect Dis. 2012;16:232-6.

10. Narciso-Schiavon JL, Schiavon L de L, Carvalho-Filho RJ, Sampaio JP, Batah PN, Barbosa DV, et al. Gender influence on treatment of chronic hepatitis $\mathrm{C}$ genotype 1. Rev Soc Bras Med Trop. 2010;43:217-23.

11. Silva GF, Polonio RJ, Pardini MI, Corvino SM, Henriques $\mathrm{RM}$, Peres $\mathrm{MN}$, et al. Using pegylated interferon alfa- $2 \mathrm{~b}$ and ribavirin to treat chronic hepatitis patients infected with hepatitis c virus genotype 1: are non-responders and relapsers different populations? Braz J Infect Dis. 2007; 11:554-60.
12. de Almeida PR, de Mattos AA, Amaral KM, Feltrin AA, Zamin $\mathrm{P}$, Tovo CV, et al. Treatment of hepatitis $\mathrm{C}$ with peginterferon and ribavirin in a public health program. Hepatogastroenterology. 2009;56:223-6.

13. Di Marco V, Covolo L, Calvaruso V, Levrero M, Puoti M, et al. Who is more likely to respond to dual treatment with pegylated-interferon and ribavirin for chronic hepatitis $C$ ? A gender-oriented analysis. J Viral Hepat. 2013;20:790-800.

14. Kwo PY, Lawitz EJ, McCone J, SchiffER, Vierling JM, Pound D, et al. Efficacy of boceprevir, an NS3 protease inhibitor, in combination with peginterferon alfa- $2 b$ and ribavirin in treatment-naive patients with genotype 1 hepatitis $C$ infection (SPRINT-1): an open-label, randomised, multicentre phase 2 trial. Lancet. 2010;376:705-16.

15. Jacobson IM, McHutchison JG, Dusheiko G, Di Bisceglie AM, Reddy KR, Bzowej NH, et al. Telaprevir for previously untreated chronic hepatitis $C$ virus infection. N Engl J Med. 2011;364:2405-16.

16. Poordad F, McCone J Jr., Bacon BR, Bruno S, Manns MP, Sulkowski MS, et al. Boceprevir for untreated chronic HCV genotype 1 infection. N Engl J Med. 2011;364:1195-206.

17. Crespo J, Berenguer M, Perez F, Fernandez I, Gonzalez $\mathrm{O}$, Barcena $\mathrm{R}$, et al. Lead-in period and week 8 as predictive tools for response to boceprevir therapy: a retrospective study of Spanish real clinical practice. Gastroenterol Hepatol. 2015. Pii:S0210-5705(15)00100-4.

18. Vo KP, Vutien P, Akiyama MJ, Vu VD, Ha NB, Piotrowski $\mathrm{JI}$, et al. Poor sustained virological response in a multicenter real-life cohort of chronic hepatitis $C$ patients treated with pegylated interferon and ribavirin plus telaprevir or boceprevir. Dig Dis Sci. 2015;60:1045-51.

19. Ministério da Saúde/CONITEC. Simeprevir, sofosbuvir e daclatasvir no tratamento da hepatite crônica tipo $\mathrm{C}$ e coinfecções. Brasil: Ministério da Saúde/CONITEC; 2015. 